\title{
Discussion: A non-linear approach for settlement prediction of single pile and pile groups under vertical load
}

\section{F. Castelli and E. Motta}

\section{N. Y. Wong and A. J. Whittle, Massachusetts Institute of Technology}

The authors have proposed an admirably simple formulation for modelling the non-linear vertical load-settlement response of single piles and pile groups in homogeneous soils that extends linear solutions of Randolph and Wroth ${ }^{23}$ and Randolph. ${ }^{4}$ In implementing this technique, we have found several errata in the formulation and flowchart, as summarised in Table 2.

We have also found some difficulties in reproducing the examples reported in the paper. Although the authors report limiting skin friction values, $f_{\mathrm{s}}$, for the single pile tests, they do not specify the actual limiting values of shaft resistance and base resistance forces $\left(R_{\text {slim }}\right.$ and $\left.R_{\text {blim }}\right)$ used in their calculations. It would be very helpful to give a complete table of these values (for both the single pile and pile groups). We have retrieved pile capacity information from the source references (Table 3) and found further inconsistencies with the authors' stated assumptions, as follows.

For Case $\mathrm{C},{ }^{27}$ the authors report $f_{\mathrm{s}}=23 \mathrm{kPa}$ and $q_{\mathrm{b}}=25 \mathrm{kPa}$ for a single pile, whereas Liu et al. measured $f_{\mathrm{s}}=19-24 \mathrm{kPa}$ for single piles and an average range of 16-23 $\mathrm{kPa}$ for each pile in the group. They also report $q_{\mathrm{b}}=173 \mathrm{kPa}$ for a single pile and $700 \mathrm{kPa}$ for each pile in the group test. There is no explanation given for the large change in end bearing of the group, but clearly the $q_{\mathrm{b}}$ value quoted by the authors is incorrect. The authors also report their analysis for a $3 \times 3$ pile group with spacing-diameter ratio $s / D=3$ (and $D=200 \mathrm{~mm}$ ), whereas the only $3 \times 3$ group tested by Liu et al. has $s / D=6$, with $D=100 \mathrm{~mm}$ (group G-6). After accounting for these discrepancies, we have found generally good agreement with the initial stiffness parameter $\left(K_{\mathrm{si}} D=3 \cdot 5\right)$ quoted by the authors.

For case B, we have had to artificially increase the end bearing resistance, $R_{\text {blim }}$ (compared with results reported by 0'Neill et $\mathrm{al}^{20}$ ) in order to achieve a reasonable match to the measured load-deformation curves for the pile groups. This behaviour reflects the measured softening of the shaft resistance in this very stiff, overconsolidated clay, a feature that is not captured by the proposed analysis. Again, there is good agreement with the initial stiffness parameter quoted by the authors $\left(K_{\mathrm{si}} D=24 \cdot 7\right)$ for both the single pile and pile groups.

We have found that much higher initial pile stiffness $\left(K_{\mathrm{si}} D=43\right.$ rather than $\left.6 \cdot 23\right)$ is necessary to match the measured load-settlement curve for the single pile of Case A installed in medium dense sand (Fig. 12), whereas the results for the pile group are more consistent with results presented by the authors $\left(K_{\mathrm{si}} D=7 \cdot 5\right.$ rather than $\left.6 \cdot 23\right)$. We are confident of our results, and suspect an error in the calculations reported by the authors for the single pile. The analyses imply that each pile in the group has a much lower initial stiffness than the single pile driven in this hydraulic fill material. This result is consistent with the findings of Briaud et al., ${ }^{26}$ who attributed this behaviour to effects of residual stresses from pile driving.

Authors' reply

The authors thank the discussers for their great 


\begin{tabular}{|c|c|c|c|c|c|}
\hline \multirow[t]{2}{*}{ Case study } & \multirow{2}{*}{$\begin{array}{l}\text { No. of } \\
\text { piles }\end{array}$} & \multicolumn{3}{|c|}{ Parameters obtained by discussers } & \multirow{2}{*}{$\frac{\text { Reported by authors }}{K_{\mathrm{si}} \mathrm{D}: \mathrm{MPa}}$} \\
\hline & & $R_{\text {blim: }}: M N$ & $\mathrm{R}_{\text {slim }}: \mathrm{MN}$ & $K_{\mathrm{si}} \mathrm{D}: \mathrm{MPa}$ & \\
\hline$A$ & I & 0.359 & 0.147 & $43 \cdot 0$ & $6 \cdot 23$ \\
\hline A & 5 & $|\cdot 2|$ & $1 \cdot 345$ & $7 \cdot 5$ & 6.23 \\
\hline B & I & 0.147 & 0.654 & $32 \cdot 0$ & $24 \cdot 70$ \\
\hline B & 4 & $\begin{array}{l}0.536 \\
0.900 *\end{array}$ & $\begin{array}{l}2.432 \\
2.696 \dagger\end{array}$ & $24 \cdot 7$ & $24 \cdot 70$ \\
\hline B & 9 & $\begin{array}{l}1.206 \\
1.600 *\end{array}$ & $\begin{array}{l}5.469 \\
6.067 \dagger\end{array}$ & $24 \cdot 7$ & $24 \cdot 70$ \\
\hline C & 1 & 0.0014 & $\begin{array}{l}0.027 \\
0.033+\end{array}$ & $2 \cdot 4-3 \cdot 5$ & $3 \cdot 50$ \\
\hline C & 16 & 0.088 & $\begin{array}{l}0.358 \\
0.444 \dagger\end{array}$ & $2 \cdot 8-3 \cdot 5$ & $3 \cdot 50$ \\
\hline
\end{tabular}

Note: Values of $R_{\text {slim }}$ and $R_{\text {blim }}$ are based on measured capacities reported in source papers.

$* R_{\text {blim }}$ obtained as best fit to measured load-deformation data.

$\dagger R_{\text {slim }}$ based on $n f_{s} A_{s}$, where $A_{s}$ is the surface area of each pile, $n$ the number of piles, and $f_{s}$ is quoted by the authors.

Table 3. Pile capacity and initial stiffness parameters

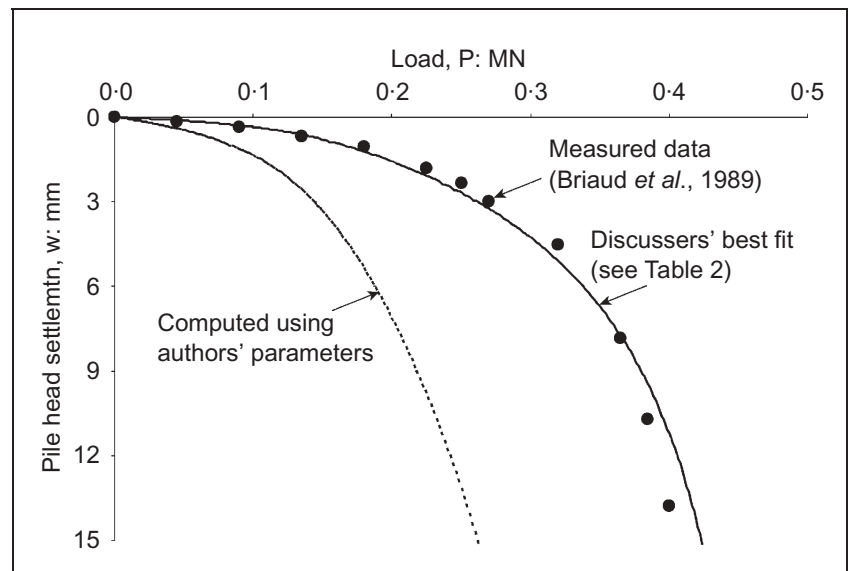

Fig. 12. Comparison of re-computed and measured behaviour for single pile, Case A

interest in the paper, and for their suggestions for the corrections of errata. The following points are added to the discussion.

For case $\mathrm{C}^{27}$ the authors wrongly reported the value $q_{\mathrm{b}}=$ $25 \mathrm{kPa}$ in the text instead of $q_{\mathrm{b}}=173 \mathrm{kPa}$, which is the actual value reported by Liu et al. However, it should be pointed out that the calculations in Fig. 9 are made for the correct value of the limit unit load at the pile base, $q_{\mathrm{b}}$.

For case $\mathrm{B}^{20}$ the authors agree with the discussers that the load-settlement curve is better reproduced if one increases the end bearing resistance for the single pile and the pile groups. Actually, in the calculations, we assumed $R_{\text {blim }}=200 \mathrm{kN}$ instead of $R_{\text {blim }}=147 \mathrm{kN}$ as reported by 0'Neill et al. For the pile groups we simply assumed a $R_{\text {blim }}$ multiplying the end bearing resistance of the single pile by the number of the piles in the group. This is in agreement with the findings of O'Neill et $a .^{20}$, which suggest an efficiency of near-unity for the pile group.

It is worthwhile to note that, for both cases B and C, the authors and the discussers agree on the evaluation of the initial stiffness to be introduced in the calculation.

For case $\mathrm{A}$ the value $K_{\mathrm{si}} D=$ 6.23 MPa reported by the authors in the paper has been wrongly attributed to the single pile. Actually this is value for the pile group. For the single pile the authors quoted the value $K_{\mathrm{si}} D=$ $2 \cdot 18 \mathrm{MPa}$. However, we suspect an error in the calculations of the discussers because of the great differences between the values of $K_{\mathrm{si}} D$ for the single pile (43 MPa) and the pile group (7.5 MPa). The diameter of the single pile is $0 \cdot 273 \mathrm{~m}$, whereas the equivalent diameter $D_{\mathrm{eq}}$ is $1.24 \mathrm{~m}$, and this suggests that the product $K_{\mathrm{si}} D$ should be larger for the pile group than for the single pile. Indeed the authors quoted a value $K_{\mathrm{si}}=80 \mathrm{MN} / \mathrm{m}^{3}$ for the single pile and $K_{\mathrm{si}}=50 \mathrm{MN} / \mathrm{m}^{3}$ for the equivalent pier.

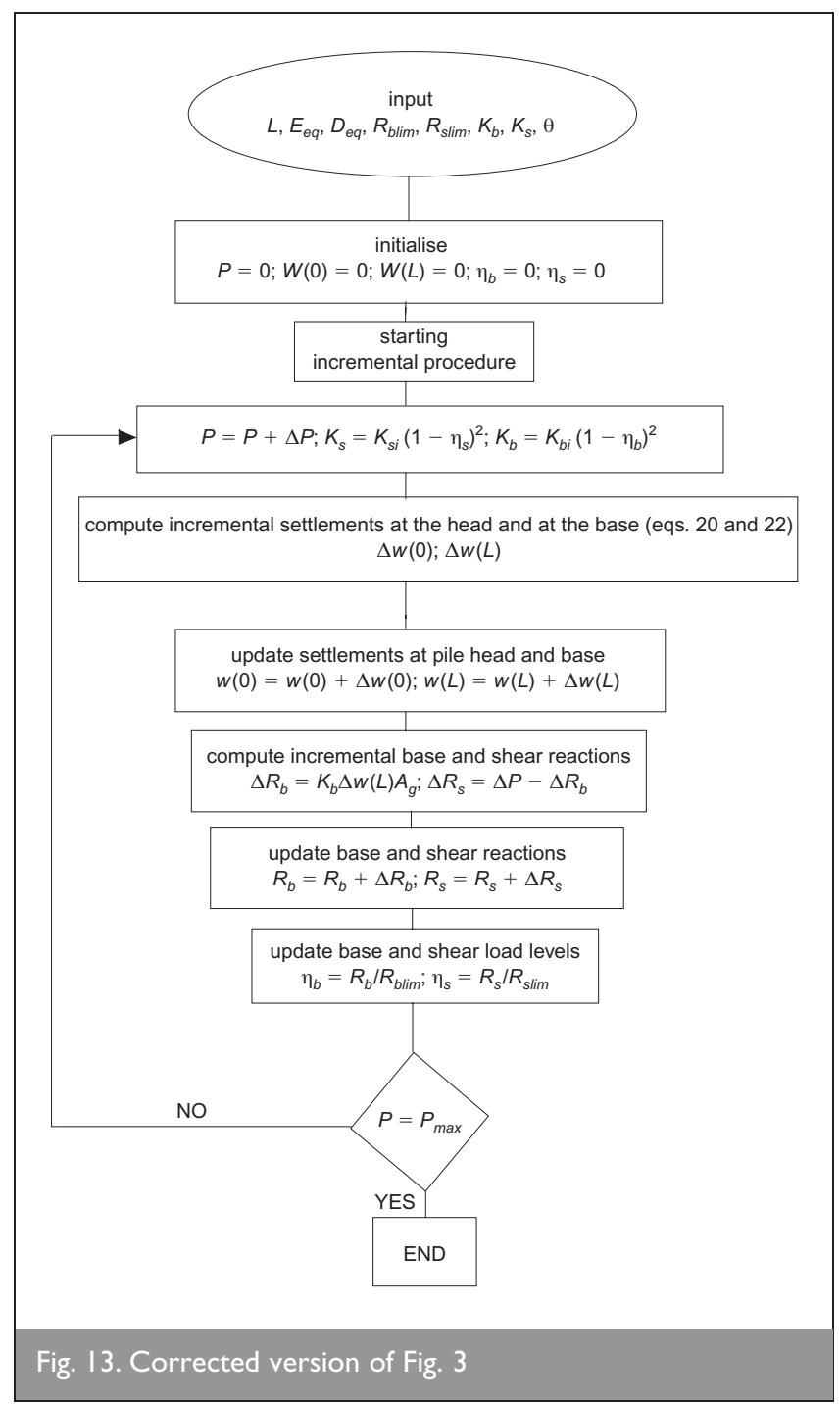


Finally, the authors are glad that the discussion was confined to the evaluation of the model parameters, as this implies that the method of analysis presented has been appreciated by the discussers.

\section{Errata}

Equations (2), (3), (19) and (22) should read as follows:

$2 \quad q=\frac{w(L)}{1 / K_{\mathrm{bi}}+w(L) / q_{\mathrm{b}}}$

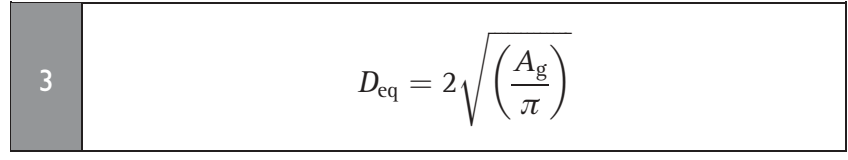

$19 \quad C_{2}=\frac{\left(1+\beta_{\mathrm{eq}}\right) \mathrm{e}^{2 \alpha^{\prime} L}}{\left(1+\beta_{\mathrm{eq}}\right) \mathrm{e}^{2 \alpha^{\prime} L}-\left(1-\beta_{\mathrm{eq}}\right)} \cdot \frac{\Delta P^{i}}{\alpha^{\prime} E_{\mathrm{eq}} A_{\mathrm{eq}}}$

$\Delta w(L)=C_{1} \mathrm{e}^{\alpha^{\prime} L}+C_{2} \mathrm{e}^{-\alpha^{\prime} L}$

In the flow chart the following lines should be corrected:

Line 4:

\begin{tabular}{l}
$K_{\mathrm{s}}=K_{\mathrm{si}}\left(1-\eta_{\mathrm{s}}\right)^{2}$ \\
$K_{\mathrm{b}}=K_{\mathrm{bi}}\left(1-\eta_{\mathrm{b}}\right)^{2}$ \\
\hline
\end{tabular}

Line 7:

$$
\begin{gathered}
\Delta R_{\mathrm{b}}=K_{\mathrm{b}} \Delta w(L) A_{\mathrm{g}} \\
\Delta R_{\mathrm{s}}=\Delta P-\Delta R_{\mathrm{b}}
\end{gathered}
$$

The corrected flowchart is therefore as shown in Figure 13. 